\title{
Article
}

\section{Does the Cosmological Expansion Change Local Dynamics?}

\author{
Marcelo Schiffer
}

check for updates

Citation: Schiffer, M. Does the Cosmological Expansion Change Local Dynamics? Symmetry 2021, 13, 1417. https://doi.org/10.3390/ sym13081417

Academic Editors: Ugur Camci, Bobomurat Ahmedov and Ashfaque H. Bokhari

Received: 6 June 2021

Accepted: 30 July 2021

Published: 3 August 2021

Publisher's Note: MDPI stays neutral with regard to jurisdictional claims in published maps and institutional affiliations.

Copyright: (C) 2021 by the author. Licensee MDPI, Basel, Switzerland. This article is an open access article distributed under the terms and conditions of the Creative Commons Attribution (CC BY) license (https:// creativecommons.org/licenses/by/ $4.0 /)$.
Physics Department, Ariel University, Ariel 40700, Israel; schiffer@ariel.ac.il

\begin{abstract}
It is a well-known fact that the Newtonian description of dynamics within Galaxies for its known matter content is in disagreement with the observations as the acceleration approaches $a_{0} \approx 1.2 \times 10^{-10} \mathrm{~m} / \mathrm{s}^{2}$ (slighter larger for clusters). Both the Dark Matter scenario and Modified Gravity Theories (MGT) fail to explain the existence of such an acceleration scale. Motivated by the closeness of the acceleration scale and the Hubble constant $\mathrm{cH}_{0} \approx 10^{-9} \mathrm{~h} \mathrm{~m} / \mathrm{s}^{2}$, we are led to analyze whether this coincidence might have a Cosmological origin for scalar-tensor and spinortensor theories by performing detailed calculations for perturbations that represent the local matter distribution on the top of the cosmological background. Then, we solve the field equations for these perturbations in a power series in the present value of the Hubble constant. As we shall see, for both theories, the power expansion contains only even powers in the Hubble constant, a fact that renders the cosmological expansion irrelevant for the local dynamics.
\end{abstract}

Keywords: cosmological expansion; Galactic dynamics; alternatives to dark matter

PACS: 04.50.Kd; 04.40.-b

\section{Introduction}

The discrepancy between the Newtonian prediction that orbital velocities within spiral Galaxies fall off $v \sim(M G / r)^{0.5}$ away from the bulk of the galactic mass distribution and observations that reveal that, in every spiral galaxy, the velocity distribution reaches a plateau as the accelerations approach the value $a_{0} \approx 1.2 \times 10^{-10} \mathrm{~m} / \mathrm{s}^{2}$. The authors in [1] led to two diametrically distinct approaches to the conundrum: (i) the Dark Matter Scenario [2] where putative non-barionic dark matter with a spherical distribution involving the disk galaxy provides the needed mass deficit to conform to the observed flat rotation curves and still adhere to the Newtonian paradigm - in this case, the Newtonian potential has a logarithmic dependence on $r$ exactly what is needed to provide the flat rotation curves; (ii) the Mond Scenario [3,4] in which the relation between the acceleration and Newtonian gravitational potential is given by

$$
\vec{\nabla} \Phi_{N}=-\mu\left(a / a_{0}\right) \vec{a}
$$

where $\mu(x)$ is a function such that $\mu(x) \rightarrow 1$ as $x>>1$ to recover the Newtonian limit and $\mu(x) \rightarrow x$ as $x<<1$ to reproduce the flat rotation curves of galaxies. One of the immediate consequences of this approach is the automatic reproduction of the Tully-Fisher Law that states the galaxy luminosity of the galaxy scales as $L \sim v^{4}$, where $v$ is the orbital velocity away from the mass distribution, provided that Luminosity tracks the Mass. The defenders of Mond claim that in order for the dark matter paradigm to conform to the Tully-Fisher law, a very precise (and quite unreasonable) fine-tuning between the halo distribution and the observed mass distribution in the galactic disk is required [5].

The MOND paradigm evolved into a relativistic equation TeVeS [6], involving the metric, a scalar and a vector field phrased in terms of a Lagrangian principle. The theory is very successful in reproducing the rotation curves in spiral Galaxies but is at odds with observed background radiation anisotropies [7]. Furthermore, TeVeS is in blatant 
disagreement with weak lensing observations. The latter is made particularly transparent by the Bullet Cluster lensing observations [8,9].

While the dark matter paradigm cannot explain the existence of the transition acceleration scale $a_{0}$, in TeVeS, it enters as a God-Given parameter in the Lagrangian. Neither one of these possibilities is theoretically acceptable. Intriguingly, $a_{0}$ comes very close to $c H_{0} \approx h 10^{-9} \mathrm{~m} / \mathrm{s}^{2}$ and raises the question of whether the change on the dynamical behavior has a cosmological origin. This avenue was exploited to some degree in the past $[10,11]$.

According to Birkhoff's theorem, in Einstein's theory, the gravitational field of a spherical symmetric mass, so the cosmological expansion cannot play any role in the local dynamics. A gauge vector field is likewise of no avail; by Gauss' theorem, it also depends upon the internal configuration. Thus, if the Cosmological expansion is to "leak" into the Galactic dynamics, scalar, spinors or non-gauge vector fields must be called for.

In this paper, we deal with a Brans-Dicke theory and carefully write down the field equations for linearized perturbations on the top of the cosmological background. Cassini data limit $\omega>4 \times 10^{4}$, which is not very generous for the Brans-Dicke theory but could well be enough to explain the dynamical changes at galactic scales or larger.

In the next section, we shall write down the field equations for the background metric and the perturbations that arise from the cosmological expansion as a series in powers of the Hubble constant. We decompose these perturbations as scalar, vector and tensor fields, as it is usually done for linear gravitational perturbations. These equations represent corrections of the dynamical equations and contain correction terms in powers of $H_{0}$. The field equations are then solved perturbatively in powers of $H_{0}$. The gravitational potential turns out to contain only even powers of $H_{0}$, and we expand it up to $H_{0}^{4}$. It turns out that all corrections are way too small to play any role in the local dynamics. Then, in the following section, we study a massless spinor field and show that, in this case, there are also no linear corrections in $H_{0}$. In the concluding remarks, we discuss that there is no a priori reason for the absence of odd powers in the Hubble constant and explore the prospects of a linear term in $H_{0}$ and in the expansion. We discuss that such terms do not resolve the conundrum. Then, we consider the possibility of an expansion of the Newtonian potential in half integer powers of the Hubble constant. Such an expansion predicts flat rotation curves and Tully-Fisher's Law.

\section{Brans-Dicke Theory}

Brans-Dicke theory is defined by the equations of motion

$$
\square \phi=\frac{8 \pi}{3+2 \omega} T^{M}
$$

and

$$
G_{a b}=8 \pi\left(\frac{T_{a b}^{M}}{\phi}+T_{a b}^{\phi}\right)
$$

where

$$
\begin{aligned}
T_{a b}^{\phi} & =\frac{\omega}{8 \pi \phi^{2}}\left(\nabla_{a} \phi \nabla_{b} \phi-\frac{1}{2} g_{a b} \nabla_{c} \phi \nabla^{c} \phi\right) \\
& +\frac{1}{8 \pi \phi}\left(\nabla_{a} \nabla_{b} \phi-g_{a b} \square \phi\right),
\end{aligned}
$$

and the matter and vacuum energy distributions are represented by

$$
T_{a b}^{M}=(p+\rho) V_{a} V_{b}+p g_{a b},
$$


where $p_{M}=0$ and $p_{\Lambda}=-\rho_{\Lambda}$ for the present state of the Universe. Consequently, $T^{M}=-\left(\rho_{M}+4 \rho_{\Lambda}\right)$. For future reference, we recall that

$$
\square \phi=-\ddot{\phi}-3 \frac{\dot{a}}{a} \dot{\phi}
$$

We wish to construct the field perturbations on the top of a cosmological background for the Brans-Dicke theory; they represent the local matter distribution. First things first, we start by solving the equations for the background fields. In the absence of any dimensional parameter, we assume that for a short time interval (the observation time),

$$
\frac{\dot{a}}{a}=H \rightarrow \frac{\dot{\phi}}{\phi}=\eta H
$$

for some dimensionless $\eta \sim \mathcal{O}(1)$. Then, with this parametrization

$$
T_{00}^{\phi}=\frac{\eta H^{2}}{16 \pi}(\omega \eta-6)
$$

and

$$
T_{\alpha \beta}^{\phi}=\frac{\eta H^{2}}{8 \pi}\left(\frac{\omega \eta}{2}+\frac{\dot{H}}{H^{2}}+2+\eta\right) a^{2} \delta_{\alpha \beta} .
$$

We identify the energy density and the pressure exerted by the field as

$$
\begin{aligned}
& \rho_{\phi}=\frac{\eta H^{2} \phi}{16 \pi}(\omega \eta-6) \\
& p_{\phi}=\frac{\eta H^{2} \phi}{8 \pi}\left(\frac{\omega \eta}{2}+\frac{\dot{H}}{H^{2}}+2+\eta\right) .
\end{aligned}
$$

Defining, as usual, $\rho_{c}=3 H^{2} \phi / 8 \pi$ and $\Omega_{X}=\rho_{X} / \rho_{c}$ from Friedman's equations

$$
\Omega_{M}+\Omega_{\Lambda}+\frac{\omega \eta^{2}}{6}-\eta=0
$$

and

$$
\frac{\dot{H}}{H^{2}}=\frac{3\left(\Omega_{\Lambda}-1\right)-\omega \eta^{2} / 2}{2+\eta}-\eta
$$

The field equation for the Brans-Dicke field yields

$$
\frac{\dot{H}}{H^{2}}=3 \frac{\Omega_{M}+4 \Omega_{\Lambda}}{(2 \omega+3) \eta}-3-\eta .
$$

Aiming to solve the perturbed equations, we display Einstein's equations in a more convenient form

$$
R_{a b}=8 \pi\left(\frac{S_{a b}^{M}}{\phi}+S_{a b}^{\phi}\right)
$$

where

$$
S_{a b}^{M}=(p+\rho) V_{a} V_{b}+\frac{\rho-p}{2} g_{a b}
$$

and

$$
S_{a b}^{\phi}=\frac{\omega}{8 \pi \phi^{2}}\left(\nabla_{a} \phi \nabla_{b} \phi\right)+\frac{1}{8 \pi \phi}\left(\nabla_{a} \nabla_{b} \phi+\frac{1}{2} g_{a b} \square \phi\right) .
$$

There are two relevant coordinate systems: the $r$-frame ( $r^{a}$ coordinates), which locally attached to the local mass distribution, and the $x$-frame ( $x^{a}$ coordinates), which is the cosmological co-moving frame, with $r^{\alpha}=a(t) x^{\alpha}$. The $r$-frame is the physically meaningful frame for local dynamics, but the $x$-frame turns out to be much more convenient for 
performing calculations. Accordingly, we construct static local disturbances in the $r$-frame (we are not interested in galactic evolution), make a coordinate transformation to the $x$-frame and perform calculations, obtaining the perturbed fields. Then, we transform them back to the $r$-frame. Let $h_{a b}(\vec{r})$ represent the static metric perturbations in the $r$-frame, then the line element is

$$
d s^{2}=\left(g_{a b}^{(0)}+h_{a b}(\vec{r})\right) d r^{a} d r^{b},
$$

where $d r^{0}=d t, g_{a b}^{(0)}$ is the cosmological smooth background. Under a ' $r^{\prime}$ to ' $x$ ' coordinate transformation, the line element perturbation looks

$$
\begin{aligned}
h_{a b}(\vec{r}) d r^{a} d r^{b}= & {\left[h_{00}+2 H h_{0 \alpha} r^{\alpha}+H^{2} h_{\alpha \beta} r^{\alpha} r^{\beta}\right] d t^{2}+2 a\left[h_{0 \alpha}+H h_{\alpha \beta} r^{\beta}\right] d x^{\alpha} d t+a^{2} h_{\alpha \beta} d x^{\alpha} d x^{\beta}, } \\
& \text { where } H=\dot{a} / a, \text { and we recall that } h_{a b}(\vec{r})=h_{a b}(a \vec{x}) .
\end{aligned}
$$

Inspecting this form, we express the perturbed metric in the $x$-frame $\tilde{h}_{a b}$ in the form:

$$
\begin{aligned}
& \tilde{h}_{00}=\psi(a \vec{x}) ; \\
& \tilde{h}_{0 \alpha}=a W_{\alpha}(a \vec{x}) ; \\
& \tilde{h}_{\alpha \beta}=a^{2} f_{\alpha \beta}(a \vec{x}),
\end{aligned}
$$

where $\psi, W_{\alpha}$ and $f_{\alpha \beta}$ are to be regarded as scalar, vector and tensor fields of a flat threedimensional space. It is reasonable to assume that the global space curvature is unimportant on a local scale; thus, locally, we take $g_{\alpha \beta}^{(0)}=a^{2} \delta_{\alpha \beta}$. Similarly, the perturbation of the scalar field is static in the physical frame, $\phi+\delta \phi=\phi\left[1+\xi\left(a x^{\alpha}\right)\right]$.

We represent the local mass distribution as a disturbance of the global smooth distribution. In this case, $\delta p$ stands for the pressure and $\delta \rho$ the mass density of the local matter distribution. Locally, $\delta p=0$ and $\delta \rho=\rho_{G}$, which is the local Galactic mass distribution. There is still one missing field $u_{a}$, the difference between the velocity of locally static observer in the $r$-frame with respect to a cosmological co-moving observer. For a static observer in the local frame, $x^{\alpha}=a^{-1} r^{\alpha}$ with constant $r^{\alpha}$. Thus, the corresponding velocity in the $x$-frame is:

$$
V_{G}^{a}=\frac{(1 ;-H \vec{x})}{\sqrt{1-H^{2} a^{2} x^{2}}} \approx(1,-H \vec{x})
$$

Recalling that $V^{b}$ is the velocity of the cosmological co-moving observer. Clearly,

$$
u_{a}=g_{a b}\left(V_{G}^{b}-V^{b}\right)+\tilde{h}_{a b} V^{b}
$$

or

$$
u_{a}=(0,-a H \vec{r})+\tilde{h}_{a 0}=\left(\psi, a\left(-H r^{\alpha}+W_{\alpha}\right)\right) .
$$

Preparing the ground for calculating the perturbations of the field equations, we first evaluate

$$
\delta\left(\nabla_{a} \nabla_{b} \phi\right)=\xi \nabla_{a} \nabla_{b} \phi+\phi \nabla_{a} \nabla_{b} \xi+\nabla_{a} \xi \nabla_{b} \phi+\nabla_{b} \xi \nabla_{a} \phi-\gamma_{a b}^{c} \phi_{c}
$$

where

$$
\gamma_{a b}^{c}=\delta \Gamma_{a b}^{c}=\frac{1}{2}\left(\nabla_{b} \tilde{h}_{a}^{c}+\nabla_{a} \tilde{h}_{b}^{c}-\nabla^{c} \tilde{h}_{a b}\right),
$$

and, consequently,

$$
\delta(\square \phi)=\xi \square \phi+\phi \square \xi+2 \nabla_{c} \xi \nabla^{c} \phi-\tilde{h}^{c d} \nabla_{c} \nabla_{d} \phi-\gamma^{c} \phi_{c}
$$

with $\gamma^{c}=g^{a b} \gamma_{a b}^{c}$. We adopt the Lorentz gauge condition,

$$
\nabla_{c} \tilde{h}_{a}^{c}-\frac{1}{2} \nabla_{a} \tilde{h}=0
$$


in which case

$$
\gamma^{c}=g^{a b} \gamma_{a b}^{c}=0,
$$

and simply drop the last term in Equation (27). We can express this gauge condition in terms of the effective 3D-fields:

$$
\begin{aligned}
& \frac{1}{a} W_{\alpha, \alpha}=\frac{1}{2}(\dot{f}+\dot{\psi})+H(f+3 \psi) \\
& \frac{1}{a} f_{\alpha \beta, \beta}=\dot{W}_{\alpha}+4 H W_{\alpha}+\frac{1}{2 a}(f-\psi)_{, \alpha} .
\end{aligned}
$$

The field equations governing the local scalar field is

$$
\xi \square \phi+\phi \square \xi+2 \nabla^{a} \xi \nabla_{a} \phi-\tilde{h}^{a b} \nabla_{a} \nabla_{b} \phi=\frac{8 \pi}{2 \omega+3} \delta T .
$$

However,

$$
\tilde{h}^{c d} \nabla_{c} \nabla_{d} \phi=\tilde{h}^{00} \ddot{\phi}-\tilde{h}^{\alpha \beta} \Gamma_{\alpha \beta}^{0} \dot{\phi}=-\psi \square \phi-3 H \dot{\phi} \psi-H \dot{\phi} f \quad,
$$

where $f \equiv \sum_{\alpha} f_{\alpha \alpha}$. Then,

$$
(f+\psi) \square \phi+\phi \square \xi-2 \dot{\phi} \dot{\xi}+H(f+3 \psi) \dot{\phi}=\frac{8 \pi}{2 \omega+3} \delta T .
$$

Clearly , $\delta T=\delta(3 p-\rho)=-\rho_{G}$ is the local energy already discussed. From Equation (2),

$$
\begin{aligned}
& (f+\psi) \frac{8 \pi}{(2 \omega+3) \phi}(-\rho+3 p)+\square \xi-2 \frac{\dot{\phi}}{\phi} \dot{\xi} \\
+ & H(f+3 \psi) \frac{\dot{\phi}}{\phi}=-\frac{8 \pi}{(2 \omega+3) \phi} \rho_{G} .
\end{aligned}
$$

We translate back our equations in terms of $r$-frame variables. In contrast to the co-moving derivative $\xi_{, \alpha}=\partial \xi / \partial x^{\alpha}$, we define the local derivative $\partial_{\alpha} \xi=\partial \xi / \partial r^{\alpha}$. Then,

$$
\left[\xi\left(a x^{\alpha}\right)\right]_{, \alpha}=a \partial_{\alpha}\left[\xi\left(r^{\alpha}\right)\right]
$$

and as the rule of the thumb we automatically replace everywhere $\partial / \partial x^{\alpha} \rightarrow a \partial / \partial r^{\alpha}$. Furthermore

$$
\frac{\partial \xi(a \vec{x})}{\partial t}=H \vec{r} \cdot \vec{\partial} \xi
$$

Then

$$
\square \xi(a \vec{x})=\left(\delta_{\alpha \beta}-H^{2} r_{\alpha} r_{\beta}\right) \partial_{\alpha} \partial_{\beta} \xi-4 H^{2} \vec{r} \cdot \vec{\nabla} \xi .
$$

With the replacement

$$
\dot{\phi} / \phi \rightarrow \eta H \quad ; \quad \phi^{-1} \rightarrow G \quad \text { and } 8 \pi \rho_{c} / \phi \rightarrow 3 H^{2},
$$

the scalar field equation (Equation (34)) looks like the following in its final form

$$
H^{2}\left[\frac{3}{(2 \omega+3)}\left(\Omega_{M}+4 \Omega_{\Lambda}\right)(\xi+\psi)-(f+3 \psi) \eta+2(\eta+2) \vec{r} \cdot \vec{\partial} \xi\right]-\left(\delta_{\alpha \beta}-H^{2} r^{\alpha} r^{\beta}\right) \partial_{\alpha} \partial_{\beta} \xi=\frac{8 \pi G}{2 \omega+3} \rho_{G}
$$

The field equations for the gravitational field are given by the linear perturbations of Einstein's equations:

$$
\delta R_{a b}=8 \pi \delta S_{a b}
$$


where

$$
\delta S_{a b} \equiv \frac{\delta S_{a b}^{M}-\xi S_{a b}^{M}}{\phi}+\delta S_{a b}^{\phi} .
$$

Let us start with the lhs. We borrow from MTW [12]:

$$
\delta R_{a b}=\frac{1}{2}\left(-\nabla_{a} \nabla_{b} \tilde{h}-\nabla^{c} \nabla_{c} \tilde{h}_{a b}+\nabla^{c} \nabla_{a} \tilde{h}_{b c}+\nabla^{c} \nabla_{b} \tilde{h}_{a c}\right),
$$

and rewrite the divergence of the gauge condition (Equation (28)) in the form:

$$
\nabla_{c} \nabla_{b} \tilde{h}_{a}^{c}=\frac{1}{2} \nabla_{b} \nabla_{a} \tilde{h}+\left[\nabla_{c} \nabla_{b}-\nabla_{b} \nabla_{c}\right] \tilde{h}_{a}^{c} .
$$

With the rule for the commutation of derivates for $(1,1)$ tensors

$$
\left[\nabla_{c} \nabla_{b}-\nabla_{b} \nabla_{c}\right] \tilde{h}_{a}^{c}=R_{d b} \tilde{h}_{a}^{d}+R_{a d c b} \tilde{h}^{c d},
$$

it follows that

$$
\nabla_{c} \nabla_{b} \tilde{h}_{a}^{c}+\nabla_{c} \nabla_{a} \tilde{h}_{b}^{c}=\nabla_{b} \nabla_{a} \tilde{h}+R_{d b} \tilde{h}^{d a}+R_{d a} \tilde{h}^{d b}+2 R_{d c b}^{a} \tilde{h}^{c d},
$$

and then

$$
\delta R_{a b}=\frac{1}{2}\left(R_{d b} \tilde{h}_{a}^{d}+R_{d a} \tilde{h}_{b}^{d}+2 R_{a d c b} \tilde{h}^{c d}-\nabla^{c} \nabla_{c} \tilde{h}_{a b}\right) .
$$

This expression is quite general. For a homogenous and isotropic background, the Weyl tensor vanishes, and the Riemann tensor is entirely described by the Ricci curvature:

$$
\begin{aligned}
R_{a d c b} & =\frac{1}{2}\left(g_{a c} R_{d b}-g_{a b} R_{c d}-g_{d c} R_{b a}+g_{b d} R_{c a}\right) \\
& +\frac{1}{6} R\left(g_{a b} g_{c d}-g_{a c} g_{d b}\right) .
\end{aligned}
$$

In that case

$$
\begin{aligned}
\delta R_{a b} & =R_{c b} \tilde{h}_{a}^{c}+R_{c a} \tilde{h}_{b}^{c}-\frac{1}{2}\left(g_{a b} R_{c d} \tilde{h}^{c d}+\tilde{h} R_{a b}\right) \\
& -\frac{1}{6}\left(\tilde{h}_{a b}-g_{a b} \tilde{h}\right) R-\frac{1}{2} \nabla_{c} \nabla^{c} \tilde{h}_{a b} .
\end{aligned}
$$

Our next step is to express $\delta R_{a b}$ in terms of the fields $f_{\alpha \beta}, W_{\alpha}$ and $\psi$ according to their definitions (Equation (21)). Furthermore, we use the field equations of the unperturbed fields (Equations (16)-(18)), obtaining

$$
\begin{aligned}
\delta R_{00} & =-\left[8 \pi \frac{3 \omega p+(\omega+3) \rho}{(2 \omega+3) \phi}+\omega \frac{\dot{\phi}^{2}}{\phi^{2}}-3 H \frac{\dot{\phi}}{\phi}\right] \psi-\left[8 \pi \frac{(1+\omega / 3) \rho+\omega p}{(2 \omega+3) \phi}+\frac{\omega}{3} \frac{\dot{\phi}^{2}}{\phi^{2}}-H \frac{\dot{\phi}}{\phi}\right] f-\frac{1}{2} \nabla^{c} \nabla_{c} \tilde{h}_{00} \\
\delta R_{0 \alpha} & =-a\left[8 \pi \frac{(2+\omega / 3) \rho+3 \omega p}{(2 \omega+3) \phi}+\frac{5 \omega}{6} \frac{\dot{\phi}^{2}}{\phi^{2}}-2 H \frac{\dot{\phi}}{\phi}\right] W_{\alpha}-\frac{1}{2} \nabla^{c} \nabla_{c} \tilde{h}_{0 \alpha} \\
\delta R_{\alpha \beta} & \left.=a^{2}\left[8 \pi \frac{\left(\frac{5 \omega}{3}+2\right) \rho-\omega p}{(2 \omega+3) \phi}-2 H \frac{\dot{\phi}}{\phi}+\frac{\omega}{6} \frac{\dot{\phi}^{2}}{\phi^{2}}\right] f_{\alpha \beta}-a^{2} \delta_{\alpha \beta}\left[\frac{8 \pi}{(2 \omega+3) \phi}[(\omega / 3+1) \rho+\omega p)\right]-H \frac{\dot{\phi}}{\phi}+\frac{\omega}{3} \frac{\dot{\phi}^{2}}{\phi^{2}}\right] \psi \\
& -a^{2} \delta_{\alpha \beta}\left[\frac{8 \pi}{3 \phi} \rho-H \frac{\dot{\phi}}{\phi}+\frac{\omega}{6} \frac{\dot{\phi}^{2}}{\phi^{2}}\right] f-\frac{1}{2} \nabla^{c} \nabla_{c} \tilde{h}_{\alpha \beta} .
\end{aligned}
$$

Furthermore,

$$
\begin{aligned}
\nabla^{c} \nabla_{c} \tilde{h}_{00} & =a^{-2} \nabla^{2} \psi-\ddot{\psi}-3 H \dot{\psi}+6 H^{2} \psi-4 H a^{-1} W_{\alpha, \alpha}+2 H^{2} f \\
\nabla^{c} \nabla_{c} \tilde{h}_{\alpha 0} & =a\left[a^{-2} \nabla^{2} W_{\alpha}-\ddot{W}_{\alpha}-3 H \dot{W}_{\alpha}+6 H^{2} W_{\alpha}-2 H a^{-1} \psi_{, \alpha}-2 H a^{-1} f_{\alpha \beta, \beta}\right] \\
\nabla^{c} \nabla_{c} \tilde{h}_{\alpha \beta} & =a^{2}\left[a^{-2} \nabla^{2} f_{\alpha \beta}-\ddot{f}_{\alpha \beta}-3 H \dot{f}_{\alpha \beta}+2 H^{2} f_{\alpha \beta}-2 H a^{-1}\left(W_{\alpha, \beta}+W_{\beta, \alpha}\right)+2 H^{2} \psi \delta_{\alpha \beta}\right] .
\end{aligned}
$$


The linear variation of Equations (17) and (18) provide the source terms of the gravitational field equations:

$$
\delta S_{a b}^{M}=\rho_{M}\left(V_{a} u_{b}+V_{b} u_{a}\right)+\frac{2 \rho_{\Lambda}+\rho_{M}}{2} \tilde{h}_{a b}+\rho_{G}\left(V_{a} V_{b}+\frac{1}{2} g_{a b}\right),
$$

together with

$$
\delta S_{a b}^{\phi}=\frac{\omega+1}{8 \pi \phi}\left(\nabla_{a} \xi \nabla_{b} \phi+\nabla_{b} \xi \nabla_{a} \phi\right)+\frac{1}{8 \pi} \nabla_{a} \nabla_{b} \xi-\frac{1}{2 \phi(2 \omega+3)}\left(g_{a b} \rho_{G}+\left(\tilde{h}_{a b}-g_{a b} \xi\right)\left(4 \rho_{\Lambda}+\rho_{M}\right)\right)-\frac{1}{8 \pi} \gamma_{a b}^{0} \frac{\dot{\phi}}{\phi}
$$

Working out the components

$$
\begin{aligned}
8 \pi \delta S_{00} & =\frac{\dot{\phi}}{2 \phi} \dot{\psi}+\ddot{\xi}+2(\omega+1) \frac{\dot{\phi}}{\phi} \dot{\xi}+\frac{8 \pi}{\phi}\left[\frac{\omega+2}{2 \omega+3} \rho_{G}+\frac{(2 \omega+1) \rho_{\Lambda}-(3 \omega+5) \rho_{M}}{2 \omega+3} \psi+\frac{(2 \omega+1) \rho_{\Lambda}-(\omega+2) \rho_{M}}{2 \omega+3} \xi\right] \\
8 \pi \delta S_{\alpha 0} & =a\left[\frac{8 \pi}{\phi} \frac{(2 \omega+1) \rho_{\Lambda}-(\omega+2) \rho_{M}}{2 \omega+3}-H \frac{\dot{\phi}}{\phi}\right] W_{\alpha}+a \frac{8 \pi}{\phi} \rho_{M} H r_{\alpha}+\left[(\omega+1) \frac{\dot{\phi}}{\phi}-H\right] \xi, \alpha+\dot{\xi}, \alpha+\frac{\dot{\phi}}{2 \phi} \psi, \alpha \\
8 \pi \delta S_{\alpha \beta} & =a^{2}\left[\frac{8 \pi}{\phi} \frac{(2 \omega+1) \rho_{\Lambda}+(\omega+1) \rho_{M}}{2 \omega+3}-H \frac{\dot{\phi}}{\phi}\right] f_{\alpha \beta}+a \frac{\dot{\phi}}{2 \phi}\left(W_{\alpha, \beta}+W_{\beta, \alpha}\right) \\
& -a^{2} \frac{\dot{\phi}}{2 \phi} \dot{f}_{\alpha \beta}+a^{2}\left[\frac{8 \pi}{\phi} \frac{\omega+1}{2 \omega+3} \rho_{G}-\frac{8 \pi}{\phi} \frac{(\omega+1) \rho_{M}+(2 \omega+1) \rho_{\Lambda}}{2 \omega+3} \xi-H \dot{\xi}-H \frac{\dot{\phi}}{\phi} \psi\right] \delta_{\alpha \beta}+\xi, \alpha \beta
\end{aligned}
$$

We shall put all the pieces together, Equations (51)-(53) with Equations (54)-(56) and (59). We use the gauge conditions (Equation (30)) and the replacements (36), (38). The 'scalar equation' that arises from the 00 component is

$$
\begin{aligned}
& H^{2}(A \psi+B f+C \xi)+H^{2}\left(3-\frac{1}{2} \eta\right) \vec{r} \cdot \vec{\partial} \psi+H^{2} \vec{r} \cdot \vec{\partial} f-H^{2}(2(\omega+1) \eta+1) \vec{r} \cdot \vec{\partial} \xi-H^{2} r_{\alpha} r_{\beta} \partial_{\alpha} \partial_{\beta} \xi \\
- & \frac{1}{2}\left(\delta_{\alpha \beta}-H^{2} r_{\alpha} r_{\beta}\right) \partial_{\alpha} \partial_{\beta} \psi=\frac{\omega+2}{2 \omega+3} 8 \pi G \rho_{G},
\end{aligned}
$$

while the vector equation that arises from the $0 \alpha$ component is

$$
H^{2} D W_{\alpha}-\frac{1}{2}\left(\delta_{\beta \gamma}-H^{2} r_{\beta} r_{\gamma}\right) \partial_{\beta} \partial_{\gamma} W_{\alpha}+3 H^{2} \vec{r} \cdot \vec{\partial} W_{\alpha}+\frac{1}{2} H \partial_{\alpha} f+\frac{1-\eta}{2} H \partial_{\alpha} \psi-H(\omega+1) \eta \partial_{\alpha} \xi-H \vec{r} \cdot \vec{\partial} \partial_{\alpha} \xi=3 \Omega_{M} H^{3} r_{\alpha}
$$

Last, the tensor equation from the $\alpha \beta$ component can be simplified with the aid of Friedman's equation (Equation (13)):

$$
-H^{2} f_{\alpha \beta}+H^{2}(P \psi+Q \xi \tilde{\xi}+\vec{\gamma} \cdot \vec{\partial} \xi) \delta_{\alpha \beta}+H\left(\frac{2-\eta}{2}\right)\left(\partial_{\alpha} W_{\beta}+\partial_{\beta} W_{\alpha}\right)+\left(\frac{\eta}{2}+2\right) H^{2} \vec{r} \cdot \vec{\partial}_{\alpha \beta}-\partial_{\alpha} \partial_{\beta} \tilde{\zeta}-\frac{1}{2}\left(\delta_{\mu v}-H^{2} r_{\mu} r_{v}\right) \partial_{\mu} \partial_{v} f_{\alpha \beta}=\frac{\omega+1}{2 \omega+3} 8 \pi G \rho_{G} \delta_{\alpha \beta},
$$

where we define the numerical coefficients coefficients:

$$
\begin{aligned}
& A=6 \frac{(\omega+1) \Omega_{M}-2 \Omega_{\Lambda}}{2 \omega+3}-\omega \eta^{2}+3(\eta+1) ; \\
& B=-\frac{(\omega+3) \Omega_{M}+(3-2 \omega) \Omega_{\Lambda}}{2 \omega+3}-\frac{\omega \eta^{2}}{3}+\eta+1 ; \\
& C=3 \frac{(\omega+2) \Omega_{M}-(2 \omega+1) \Omega_{\Lambda}}{2 \omega+3} ; \\
& D=\frac{2 \omega \Omega_{M}+(2 \omega-9) \Omega_{\Lambda}}{2 \omega+3}-5 \frac{\omega \eta^{2}}{6}+3 \eta-1 ; \\
& P=\frac{(\omega+3) \Omega_{M}-(2 \omega-3) \Omega_{\Lambda}}{2 \omega+3}-2 \eta+\frac{\omega}{3} \eta^{2}+1 ; \\
& Q=3 \frac{(\omega+1) \Omega_{M}+(2 \omega+1) \Omega_{\Lambda}}{2 \omega+3}
\end{aligned}
$$




\section{Solving the Equations by Perturbation}

At this stage, a remark of caution is in order. Albeit the perturbation fields $\psi, W_{\alpha}$ and $f_{\alpha \beta}$ stand for $\tilde{h}_{\alpha \beta}$ and are functions of the local coordinate $\vec{r}$, they are still metric perturbations in the $x$-frame (see Equations (20) and (21)):

$$
d s^{2}=\tilde{g}_{a b}^{(0)} d x^{a} d x^{b}+\psi d t^{2}+2 a W_{\alpha} d x^{\alpha} d t+a^{2} f_{\alpha \beta} d x^{\alpha} d x^{\beta} .
$$

Transforming back to the $r$-frame:

$$
d s^{2}=g_{a b}^{(0)} d r^{a} d r^{b}-\left(\psi+2 H W_{\alpha} r^{\alpha}-H^{2} f_{\alpha \beta} r^{\alpha} r^{\beta}\right) d t^{2}+2\left(W_{\alpha}-H f_{\alpha \beta} r^{\alpha}\right) d r^{\alpha} d t+f_{\alpha \beta} d r^{\alpha} d r^{\beta} .
$$

Clearly,

$$
\begin{aligned}
& h_{00}=-\psi-2 H W_{\alpha} r^{\alpha}+H^{2} f_{\alpha \beta} r^{\alpha} r^{\beta} \\
& h_{0 \alpha}=W_{\alpha}-H f_{\alpha \beta} r^{\beta} \\
& h_{\alpha \beta}=f_{\alpha \beta} .
\end{aligned}
$$

We shall consider spherically symmetric configurations alone. In this case,

$$
W_{\alpha}=W(r) \hat{r}_{\alpha} \quad ; \quad f_{\alpha \beta}=A(r) \delta_{\alpha \beta}+B(r) \hat{r}_{\alpha} \hat{r}_{\beta} \quad,
$$

where $A, B$ and $W$ are 'scalar fields'. Then,

$$
\begin{aligned}
& \partial^{2} W_{\alpha}=\left(\partial^{2} W-2 \frac{W}{r^{2}}\right) \hat{r}_{\alpha} \\
& \partial^{2} f_{\alpha \beta}=\left(\partial^{2} A+\frac{2 B}{r^{2}}\right) \delta_{\alpha \beta}+\left(\partial^{2} B-\frac{6 B}{r^{2}}\right) \hat{r}_{\alpha} \hat{r}_{\beta},
\end{aligned}
$$

and also

$$
\begin{aligned}
& \partial_{\alpha} W_{\alpha}=W^{\prime}+\frac{2 W}{r} \\
& \partial_{\beta} f_{\alpha \beta}=\left(A^{\prime}+B^{\prime}+\frac{2 B}{r}\right) \hat{r}_{\alpha} .
\end{aligned}
$$

Next, we introduce these expressions into the their corresponding Equations (64)-(66) and solve them pertubatively in powers of $H$. The zeroth order satisfying the gauge conditions is

$$
\begin{aligned}
\xi^{(0)} & =\frac{1}{2 \omega+3} \frac{2 M G}{r} \\
\psi^{(0)} & =\frac{\omega+2}{2 \omega+3} \frac{4 G M}{r} \\
f_{\alpha \beta}^{(0)} & =\frac{2 G M}{r} \delta_{\alpha \beta}+\frac{\omega+1}{2 \omega+3} \frac{2 M G}{r} \hat{r}_{\alpha} \hat{r}_{\beta} \\
W_{\alpha}^{(0)} & =0 .
\end{aligned}
$$

The easiest way of getting $W$ is by substituting the previous results into the gauge condition in Equation (30). From now on, we drop numerical coefficients, then

$$
\partial_{\alpha} W_{\alpha}^{(1)} \sim \frac{M G}{r}
$$

and by virtue of Equation (79), it follows that $W \sim M G$ and no r-dependence, and then

$$
W_{\alpha}^{(1)} \sim M G \hat{r}^{\alpha}
$$


To the second order, we have

$$
\begin{aligned}
& \partial^{2} \psi^{(2)}=2\left(A \psi^{(0)}+B f^{(0)}+C \xi^{(0)}\right)+2\left(3-\frac{1}{2} \eta\right) \vec{r} \cdot \vec{\partial} \psi^{(0)}+2 \vec{r} \cdot \vec{\partial} f^{(0)}-2(2(\omega+1) \eta+1) \vec{r} \cdot \vec{\partial} \xi^{(0)}+r_{\alpha} r_{\beta} \partial_{\alpha} \partial_{\beta}\left(\psi^{(0)}-2 \xi^{(0)}\right) \\
& \partial^{2} f_{\alpha \beta}^{(2)}=2 f_{\alpha \beta}^{(0)}+2\left(P \psi^{(0)}+Q \xi^{(0)}+\vec{r} \cdot \vec{\partial} \xi^{(0)}\right) \delta_{\alpha \beta}+(2-\eta)\left(\partial_{\alpha} W_{\beta}^{(1)}+\partial_{\beta} W_{\alpha}^{(1)}\right)+(\eta+4) \vec{r} \cdot \vec{\partial} f_{\alpha \beta}^{(0)}-2 \partial_{\alpha} \partial_{\beta} \xi^{(0)}+r_{\mu} r_{\nu} \partial_{\mu} \partial_{\nu} f_{\alpha \beta}^{(0)} \\
& \text { and } \\
& \partial^{2} \xi^{(2)}=\frac{3}{(2 \omega+3)}\left(\Omega_{M}+4 \Omega_{\Lambda}\right)\left(\xi^{(0)}+\psi^{(0)}\right)-\eta\left(f^{(0)}+3 \psi^{(0)}\right)+2(\eta+2) \vec{r} \cdot \vec{\partial} \xi^{(0)}+r^{\alpha} r^{\beta} \partial_{\alpha} \partial_{\beta} \xi^{(0)} \\
& \text { whose solution is } \\
& \begin{array}{l}
\psi^{(2)} \sim \operatorname{MGr} \\
\xi^{(2)} \sim \operatorname{MGr} \\
f_{\alpha \beta}^{(2)} \sim \operatorname{MGr}\left(\delta_{\alpha \beta}+\hat{r}_{\alpha} \hat{r}_{\beta}\right)
\end{array}
\end{aligned}
$$

at higher orders

$$
\begin{aligned}
\partial^{2} W^{(3)} & =2 W_{\alpha}^{(1)}+r_{\beta} r_{\gamma} \partial_{\alpha} \partial_{\beta} W^{(1)}+6 \vec{r} \cdot \vec{\partial} W_{\alpha}^{(1)}+\partial_{\alpha} f^{(2)}+(1-\eta) \partial_{\alpha} \psi^{(2)} \\
& -2(\omega+1) \eta \partial_{\alpha} \xi^{(2)}-2 \vec{r} \cdot \vec{\partial} \partial_{\alpha} \xi^{(2)}-6 \Omega_{M} r_{\alpha} .
\end{aligned}
$$

Accordingly,

$$
W_{\alpha}^{(3)} \sim\left(M G r^{2}-r^{3}\right) \hat{r}_{\alpha}
$$

The fourth order equations for $\psi^{(4)}$ and $\xi^{(4)}$ are identical to Equations (86) and (87). Therefore,

$$
\psi^{(4)} \sim M G r^{3} ; \xi^{(4)} \sim M G r^{3}
$$

Thus, by virtue of Equation (75),

$$
\begin{aligned}
g_{00} & \sim-1+H^{2} r^{2}+\frac{\omega+2}{2 \omega+3} \frac{4 G M}{r}+H^{2} M G r \\
& -H^{4} r^{4}+H^{4} M G r^{3}+\ldots
\end{aligned}
$$

The term $H^{2} r^{2}$ arrives from the coordinate transformation from the $x$ frame to the $r$-frame (see Equation (75)). A comparison with the Newtonian potential term $G M / r$ reveals that it becomes relevant as $r^{3} \sim M G H^{-2}$ or $r \sim 400 \mathrm{kpc}$ for a typical galaxy. On the same grounds, the correction $H^{2} M G r$ becomes relevant only at the Hubble distance $r \sim H$. Notice that there are no linear terms on $H$ that could bring about relevant corrections to the local dynamics.

\section{Spinor Field}

In the Brans-Dicke theory, the lowest order in $H$ corrections of the field equations are quadratic in the Hubble constant. We wonder if a spinor field, whose energy momentum tensor contains first derivatives of the spinor field, could remedy the problem and yield larger contributions. Since we agreed not to settle the scale of $a_{0}$ through external given parameters, we concentrate on a massless particle. All non-zero momentum modes can be swept into the energy momentum tensor of the matter distribution, and the discussion is similar to that of the previous section. Nevertheless, the zero mode has no particle content and must be dealt with separately. We consider this mode as being a cosmological substrate that is deformed in the presence of a mass distribution and calculate its contribution to the energy-momentum tensor.

In a curved space-time, the Dirac equation reads

$$
\left[i \gamma^{a} e_{(a)}^{m}\left(\frac{\partial}{\partial x^{m}}+\frac{1}{4} C_{m b c} \gamma^{b} \gamma^{c}\right)-m\right] \Psi=0,
$$


where $e_{m}^{(a)} a=1, \ldots, 4$ are the four tetrads (the index in bracket is a Lorentz index, and the other one is the space-time component),

$$
g_{m n}=e_{m}^{(a)} e_{n}^{(b)} \eta_{a b}
$$

Bracketed indexes of the tetrads are raised/lowered with $\eta_{a b}$, and unbracketed indexes with the space-time metric $g_{m n}$ and $\gamma^{a}$ are the Dirac matrices:

$$
\left\{\gamma^{a}, \gamma^{b}\right\}=2 \eta^{a b}
$$

and the spin connection is defined as

$$
C_{m(a)(b)}=e_{(a)}{ }^{n} e_{(c) n ; m} .
$$

Furthermore, one defines the derivative operator

$$
D_{m}=\frac{\partial}{\partial x^{m}}+\frac{1}{4} C_{m b c} \gamma^{b} \gamma^{c}
$$

The energy momentum tensor is

$$
T_{m n}=\left(\frac{i}{4} e_{(a) m} \bar{\Psi} \gamma^{a} D_{n} \Psi+\text { c.c. }\right)+m \leftrightarrow n,
$$

where the swapping $m \leftrightarrow n$ of indexes is carried out for symmetrisation. The tetrads of the Robertson-Walker metric are diagonal:

$$
e_{0}^{(0)}=1 \quad ; \quad e_{\beta}^{(\alpha)}=a \delta_{\beta}^{\alpha},
$$

where Greek indexes run over the spatial components, and $a=a(t)$ is the cosmological radius scale. In this case, the only non-vanishing components of spin-connection are

$$
C_{\alpha 0 \beta}=-\dot{a} \delta_{\alpha \beta},
$$

after some algebra, the Dirac Equation reads

$$
\left[i\left(\frac{\partial}{\partial t}-a^{-1} \gamma^{0} \vec{\gamma} \cdot \vec{\nabla}+\frac{3 \dot{a}}{2 a}\right)-\gamma^{0} m\right] \Psi=0,
$$

where $\vec{\nabla}_{\alpha}=\partial / \partial x^{\alpha}$.

The generic solution is of the form $\Psi=\Phi(t) e^{-i \vec{k} \cdot \vec{x}}$. For a massless and zero momentum configuration, $\Psi(t)=\Psi_{0} a^{-3 / 2}$ with $\Psi_{0}$ a constant spinor. The energy-momentum components are

$$
\begin{aligned}
& T_{00}=i \frac{3 H}{4} \Psi^{\dagger} \Psi+c . c=0 \\
& T_{\alpha \beta}=-i \frac{\dot{a}}{4} \Psi^{\dagger} \Psi \delta_{\alpha \beta}+c . c=0,
\end{aligned}
$$

since $\Psi^{\dagger} \Psi$ is real. Thus, the zero mode (substrate) does not modify the cosmological dynamics.

Consider now the perturbations generated by the local gravitational field. The departure of the spinor from the cosmological background is here defined as $\Psi+\delta \Psi=$ $a^{-3 / 2}\left(\Psi_{0}+\Theta\right)$ and the tetrad variation $\delta e_{m}^{(a)}=\varepsilon_{m}^{(a)}$ such that

$$
\tilde{h}_{m n}=\varepsilon_{m}^{(a)} e_{(a) n}+\varepsilon_{n}^{(a)} e_{(a) n} .
$$


Last, we define $\sigma_{m a b}=\delta C_{m a b}$. One shows that

$$
\sigma_{m a b}=\frac{1}{2} \varepsilon^{(c) n}\left(e_{(a) n} C_{m a b}-e_{(b) n} C_{m a c}\right)+\frac{1}{2}\left(e_{(a)}^{n} \varepsilon_{(b) n ; m}-e_{(b)}^{n} \varepsilon_{(a) n ; m}\right)+\frac{1}{2} e_{(b)}^{l} e_{(a)}^{p}\left(\tilde{h}_{m p ; l}-\tilde{h}_{m l: p}\right) .
$$

Then, the perturbed Dirac equation reads

$$
\left[\left(\frac{\partial}{\partial t}-a^{-1} \gamma^{0} \vec{\gamma} \cdot \vec{\nabla}+\frac{3 \dot{a}}{2 a}\right)+i \gamma^{0} m\right] a^{-3 / 2} \Theta=-\gamma^{0}\left[\gamma^{a} \varepsilon_{(a)}^{m} \partial_{m}+\frac{1}{4}\left(\varepsilon_{(a)}^{m} \partial_{m} C_{m b c}-e_{(a)}^{m} \sigma_{m b c}\right) \gamma^{b} \gamma^{c}\right] \Psi
$$

To proceed further, we specify the perturbation of the tetrad:

$$
\begin{aligned}
2 \varepsilon_{(0) 0} & =\psi \\
\varepsilon_{(0) \alpha}+\varepsilon_{(\alpha) 0} & =W_{\alpha} \\
\varepsilon_{(\beta) \alpha}+\varepsilon_{(\alpha) \beta} & =a f_{\alpha \beta} .
\end{aligned}
$$

Since the tetrad $\varepsilon_{(0) a}$ is time-like, through a Lorentz transformation, we can eliminate all the spatial components $\varepsilon_{(0) \alpha}$. Thus, in this particular Lorentz frame, $\varepsilon_{(0) \alpha}=0$ and

$$
\varepsilon_{(0) 0}=\frac{1}{2} \psi \quad ; \quad \varepsilon_{(\alpha) 0}=W_{\alpha} \quad ; \quad \varepsilon_{(\alpha) \beta}=\frac{a}{2} f_{\alpha \beta} .
$$

Inserting these tetrads into Equation (105), yields

$$
\begin{aligned}
\sigma_{00 \alpha} & =\frac{1}{2 a} \psi, \alpha-H W_{\alpha} \\
\sigma_{0 \alpha \beta} & =\frac{1}{2 a}\left(W_{\alpha, \beta}-W_{\beta, \alpha}\right) \\
\sigma_{\alpha 0 \beta} & =\frac{a}{2}\left(a^{-1}\left(W_{\alpha, \beta}+W_{\beta, \alpha}\right)-H f_{\alpha \beta}-\dot{f}_{\alpha \beta}-H \psi \delta_{\alpha \beta}\right) \\
\sigma_{\alpha \beta \gamma} & =\frac{a}{2}\left(a^{-1}\left(f_{\alpha \beta, \gamma}-f_{\alpha \gamma, \beta}\right)+H\left(\delta_{\alpha \beta} W_{\gamma}-\delta_{\alpha \gamma} W_{\beta}\right)\right)
\end{aligned}
$$

Inserting Equations (108) and (109) into (106) while recalling the substitution $a^{-1} \partial / \partial x^{\alpha}=\partial / \partial r^{\alpha}$ leads, after some algebra, to

$$
\left[\frac{\partial}{\partial t}-\gamma^{0} \vec{\gamma} \cdot \vec{\partial}\right] \Theta=\left[\left(\frac{1}{4} \dot{f}-\frac{1}{2} \partial_{\alpha} W_{\alpha}\right)+\left(\frac{H}{2} W_{\alpha}+\frac{1}{4} \partial_{\beta} f_{\alpha \beta}\right) \gamma^{0} \gamma^{\alpha}-\frac{i}{4} \partial_{\beta} W_{\alpha} \sigma^{\alpha \beta}\right] \Psi_{0},
$$

where $\sigma^{\alpha \beta}=i\left[\gamma^{\alpha}, \gamma^{\beta}\right] / 2$ and $m=0$.

The time-dependent solution $\Theta=\theta(\vec{r}) e^{-i E t}$ is not consistent with the rhs unless $E=0$. This is in agreement with the fact that we regard $\Theta$ as a distortion of the minimum energy configuration $\Psi$ (the substrate) due to the local gravitational field. Recalling that $\partial / \partial t$ is a derivative with $\vec{x}$-constant of a function that depends on $\vec{r}$, we can replace $\partial / \partial t \rightarrow H \vec{r} \cdot \vec{\partial}$

$$
\left[H \vec{\gamma} \cdot \vec{\partial}-\gamma^{0} \vec{\gamma} \cdot \vec{\partial}\right] \Theta=\left[\left(\frac{H}{4} \vec{\gamma} \cdot \vec{\partial} f-\frac{1}{2} \partial_{\alpha} W_{\alpha}\right)+\left(\frac{H}{2} W_{\alpha}+\frac{1}{4} \partial_{\beta} f_{\alpha \beta}\right) \gamma^{0} \gamma^{\alpha}-\frac{i}{4} \partial_{\beta} W_{\alpha} \sigma^{\alpha \beta}\right] \Psi_{0} \text {. }
$$

In the spirit of the previous discussions, we solve the equation perturbatively:

$$
\Theta=\Theta^{(0)}+H \Theta^{(1)}+H^{2} \Theta^{(1)}+\cdots .
$$

As in the previous section, $W_{\alpha}$ starts at the order $\sim \mathcal{O}(H)$ (it is related to $T_{0 \alpha}$ equation, and it vanishes for a static configuration). Then to the lowest order in $H$

$$
\vec{\gamma} \cdot \vec{\partial} \Theta^{(0)}=-\frac{1}{4} \gamma^{\beta} \partial_{\alpha} f_{\alpha \beta}^{(0)} \Psi_{0}
$$

Applying $\vec{\gamma} \cdot \vec{\partial}$ on both sides

$$
\partial^{2} \Theta^{(0)}=-\frac{1}{4} \gamma^{\mu} \partial_{\mu} \gamma^{\beta} \partial_{\alpha} f_{\alpha \beta}^{(0)} \Psi_{0}
$$




$$
\begin{aligned}
& \text { whose solution is } \\
& \Theta^{(0)}=-\gamma^{\alpha} \gamma^{\beta} F_{\alpha \beta} \Psi_{0} \\
& F_{\alpha \beta}=\frac{1}{16 \pi} \int \frac{\partial_{\alpha}^{\prime} \partial_{\mu}^{\prime} f^{(0)^{\prime}} \mu \beta}{\left|\vec{r}-\vec{r}^{\prime}\right|} d^{3} r^{\prime} \\
& \vec{\gamma} \cdot \vec{\partial} \Theta^{(1)}=\left[\vec{r} \cdot \vec{\partial} \gamma^{\alpha} \gamma^{\beta} F_{\alpha \beta}+\frac{1}{4} \vec{r} \cdot \vec{\partial} f^{(0)}-\frac{1}{2} \partial_{\alpha} W_{\alpha}^{(1)}+\frac{1}{4} \partial_{\beta} f_{\alpha \beta}^{(1)} \gamma^{\alpha}-\frac{i}{4} \partial_{\beta} W_{\alpha}^{(1)} \sigma^{\alpha \beta}\right] \gamma^{0} \Psi_{0} . \\
& \delta T_{m n}=\left\{\left[\frac{i}{4} \varepsilon_{(a) m}\left(\bar{\Psi} \gamma^{a} \partial_{n} \Psi+\frac{1}{4} C_{n a b} \bar{\Psi} \gamma^{a} \gamma^{b} \gamma^{c} \Psi\right)+\frac{i}{16} e_{(a) m} \sigma_{n b c} \bar{\Psi} \gamma^{a} \gamma^{b} \gamma^{c} \Psi\right.\right. \\
& \left.\left.+\quad a^{-3 / 2} \frac{i}{4} e_{(a) m}\left(\bar{\Theta} \gamma^{a} D_{n} \Psi+\bar{\Psi} \gamma^{a} D_{n} \Theta\right)\right]+c . c\right\}+m \leftrightarrow n
\end{aligned}
$$

We are mainly interested in the $\delta T_{00}$ component. Recalling that $C_{0 a b}=0, \Psi(t)=$ $\left(a_{0} / a\right)^{3 / 2} \Psi_{0}$, we get

$$
\delta T_{00}=\left(\frac{a_{0}}{a}\right)^{3 / 2}\left[-i \frac{3 H}{4} \varepsilon_{(a) 0} \bar{\Psi}_{0} \gamma^{a} \Psi_{0}-\frac{i}{8} \sigma_{0 \beta \gamma} \bar{\Psi}_{0} \gamma^{0} \gamma^{\beta} \gamma^{\gamma} \Psi_{0}+\frac{i}{4} \sigma_{00 \alpha} \bar{\Psi}_{0} \gamma^{\alpha} \Psi_{0}+\frac{i}{2}\left(-\frac{3 H}{2} \Theta^{\dagger} \Psi_{0}+\Psi_{0}^{\dagger} \partial_{0} \Theta\right)\right]+c . c
$$

Now, $\bar{\Psi} \gamma^{a} \Psi$ is real and the current $\bar{\Psi}_{0} \gamma^{\alpha} \Psi_{0}=0$ since there is no preferred cosmological direction. Furthermore, for a spherical symmetrical configuration, $\sigma_{0 \alpha \beta}=0$ (see Equation (109)), thus

$$
\delta T_{00}=i \frac{H}{2}\left(\frac{a_{0}}{a}\right)^{3 / 2}\left(-\frac{3}{2} \Theta^{\dagger} \Psi_{0}+\vec{r} \cdot \vec{\nabla} \Psi_{0}{ }^{\dagger} \Theta\right)+c . c
$$

To the first order in $H$, we need only $\Theta_{0}$ (Equation (115)),

$$
\delta T_{00} \sim i H F_{\alpha \beta} \Psi_{0}^{\dagger} \gamma^{\alpha} \gamma^{\beta} \Psi_{0}+c c
$$

Clearly, in a spherical symmetrical configuration, $F_{\alpha \beta}$ is symmetric, thus

$$
\delta T_{00} \sim i H F \Psi_{0}^{\dagger} \Psi_{0}+c . c=0
$$

where $F=\sum_{\alpha} F_{\alpha \alpha}$. Accordingly, a spinor cannot induce a first order in $H$ correction to the Newtonian potential.

Unforseeably, none of the field theories studied in this paper can produce odd corrections in $H$ to the local gravitational fields and, therefore, cannot bring about substantial corrections to the local dynamics.

\section{Concluding Remarks}

In the lack of a general principle forbidding odd powers in $H$, it is conceivable that some field theory could bring about odd powers in the $H$-expansion. Should such a theory exist, the lowest order corrections are linear in $H$ and on dimensional grounds

$$
\psi \sim-\frac{M G}{r}+H r+H M G \ln (r)+\ldots
$$

Accordingly, the velocity profile away from the mass distribution would be

$$
v^{2} \sim \frac{M G}{r}+H r+M G H+\cdots
$$


The last term yields flat rotation curves, but compared to the Newtonian term, it only becomes relevant at scales $r_{0} \sim H^{-1}$; thus, it is meaningless. The second term gives a linearly growing velocity curve at a very much small slope such that could be mistakenly taken for a flat rotation curve at $5 \mathrm{kpc}$, but for larger scales, the velocity curve would behave as $\sqrt{r}$, which is not observed. Therefore, a theory that yields linear terms of the cosmological constant does not solve the flat rotation curves conundrum. However, a theory that contains half integer powers of $H_{0}$ should contain a term of the form $\sqrt{M G H} l n r$ in the Newtonian potential. Clearly, such a term yields flat rotation curves with $v^{4} \sim H M G$, which is precisely Tully-Fisher's law. It remains to be shown whether such a theory can be constructed. For the time being, the only satisfying theoretical paradigm for explaining flat rotation remains dark matter.

Funding: This research received no external funding.

Institutional Review Board Statement: Not applicable.

Informed Consent Statement: Not applicable.

Data Availability Statement: Not applicable.

Conflicts of Interest: The author declares no conflict of interest.

\section{References}

1. Sanders, R.H.; McGaugh, S.S. Modifed Newtonian Dynamics as an Alternative to Dark Matter. Ann. Rev. Astron. Astrophys. 2002, 40, 263-317. [CrossRef]

2. Navarro, J.F.; Frenk, C.S.; White, S.D.M. The structure of cold dark matter halos. Astrophys. J. 2006, 463, 563-575. [CrossRef]

3. Milgrom, M. A modification of the Newtonian dynamics-Implications for galaxies. Astrophys. J. 1983, 270, 371-389. [CrossRef]

4. Milgrom, M. A modification of the Newtonian dynamics as a possible alternative to the hidden mass hypothesis. Astrophys. J. 1983, 270, 365-370. [CrossRef]

5. Bekenstein, J.D. Tensor-Vector-Scalar modified gravity: From small scale to cosmology. Philos. Trans. R. Soc. Math. Phys. Eng. Sci. 2011, 369, 5003-5017. [CrossRef] [PubMed]

6. Bekenstein, J.D. Relativistic gravitation theory for the modified Newtonian dynamics paradigm. Phys. Rev. D 2004, 70, 083509. [CrossRef]

7. Skordis, C. The Tensor-Vector-Scalar theory and its cosmology-Skordis, Constantinos. Class. Quant. Gravity 2009, $26,143001$. [CrossRef]

8. Angus, G.W.; Shan, H.; Zhao, H.; Famaey, B. On the Law of Gravity, the Mass of Neutrinos and the Proof of Dark Matter. Astrophys. J. 2007, 654, L13-L16. [CrossRef]

9. Bekenstein, J.D. The modified Newtonian dynamics-MOND-and its implications for new physics. Contemp. Phys. 2006, $47,387$. [CrossRef]

10. Noerslinger, P.D.; Petrosian, V. The effect of cosmological expansion on self-gravitating ensembles of particles. Astrophys. J. 1971, 168, 1. [CrossRef]

11. Feinstein, A.; Ibánez, J.; Lazkoz, R. Disks in Expanding FRW Universes. Astrophys. J. 1998, 495, 131. [CrossRef]

12. Misner, C.W.; Kip, S. Thorne and John Archibald Wheeler. In Gravitation, 1st ed.; WH Freeman: San Francisco, CA, USA, 1973. 